\title{
Felicidad y actividad física en personas mayores
}

\section{Happiness and physical activity in the elderly}

\author{
María del Rocío Bohórquez, Macarena Lorenzo y Alfonso Javier García
}

Universidad de Sevilla, España

Disponible online 31 de agosto de 2013

\begin{abstract}
El principal objetivo de este estudio fue explorar la felicidad -presente y pasada- de personas mayores de 65 años, así como la relación que la práctica de Actividad Física pudiera tener con ella. Los participantes cumplimentaron voluntariamente un cuestionario de datos sociodemográficos, el Cuestionario de actividad Física General en versión española y la Escala de Felicidad. Los resultados muestran que la felicidad actual de los mayores es inferior a la felicidad pasada. Además, se demuestra que la práctica actual de actividad física incide en la felicidad de los mayores, siendo más felices aquellos más activos en esta etapa de su vida. Finalmente, haber estado implicado en la práctica de actividad física en algún momento del ciclo vital (en el pasado, en el presente o de manera continua) está relacionado con un nivel mayor de felicidad que haber tenido un estilo de vida sedentario, los mecanismos gracias a los cuales la práctica de actividad física tiene este papel protector de la felicidad están por determinar. Este papel protector de la actividad física ha de ser tenido en cuenta en el diseño de los programas de promoción e intervención en el envejecimiento activo y positivo de la población.
\end{abstract}

Palabras clave: Felicidad; Bienestar; Ejercicio Físico; Vejez.

The overall objective of this study was to explore the relationship between past and present happiness and physical activity among people over 65 years old. Participants voluntarily completed a sociodemographic questionnaire, the the Spanish version of the Global Physical Activity Questionnaire and the Happiness Scale. The results show that the participants are less happy now than they were in the past. Furthermore, the current practice of physical activity leads to an increase in happiness, the happiest individuals being those who are the most active at this stage of life. Finally, being involved in physical activity at some point in the life cycle (past, present, or continuously) is associated with a higher level of happiness than that associated with a sedentary lifestyle. The mechanisms involved in the protective role of happiness remain to be determined. The protective role of physical activity has to be taken into account in the design of programs and interventions promoting active and positive aging.

Key words: Happiness; Well-being; Exercise; Old Age.

Correspondencia: Maa Rocío Bohórquez Gómez-Millán; Av. Manuel Siurot 8. 41013, Sevilla. E-mail: rociobohorquez@us.es. E-mail de los otros autores: Macarena Lorenzo: macarena.lorenzo.fernandez@gmail.co; Alfonso Javier García: alfonsoj@us.es. 
Calidad de vida, bienestar subjetivo y felicidad son términos que, aunque a menudo han sido utilizados indistintamente, denotan significados diferentes (Veenhomen, 2000). Atendiendo al modelo de bienestar subjetivo de Diener (1984; Diener y Suh, 1998), el bienestar subjetivo incluiría respuestas emocionales -balance afectivo- y juicios globales de la satisfacción con la vida, ambos en relación a dominios concretos (familia, trabajo, etcétera). Desde esta óptica, la felicidad sería un resultado en la evaluación del bienestar subjetivo caracterizado por un balance afectivo positivo y un juicio global de satisfacción con la vida en el dominio del yo (Diener y Suh 1998).

Dos tipos de factores han sido evaluados en su relación con el bienestar subjetivo: de un lado variables sociodemográficas y de otro, factores psicosociales. En lo relativo a las variables sociodemográficas se ha abordado la influencia de la edad (McMahan y Estes, 2012; Pinquart, 2001), el género (Pinquart y Sorensen, 2001), la raza (Liang, Lawrence y Bollen, 1987; Mckenzie y Campbell, 1987), el estatus marital (HaringHidore, Stock, Okun y Witter, 1985; Waldinger y Schultz, 2010), las relaciones familiares (Diener y Suh, 1998; Pinquard y Sorensen, 2000), las relaciones sociales (Pinquart y Sorensen, 2000), la situación laboral (VanPraag, Frijters y Ferrer-i-Carbonell, 2003) o el estado de salud (Okun, Stock, Haring y Witter, 1984; VanPraaget al., 2003). A pesar de este amplio rango de factores estudiados, se ha postulado una escasa influencia de las variables sociodemográficas en la percepción del bienestar subjetivo (Diener y Suh, 1998; Diener, Suh, Lucas y Smith, 1999).

Por contra, los factores psicosociales han sido identificados como la mayor fuente de variabilidad en relación al bienestar subjetivo (Godoy-Izquierdo, Lara, Vázquez, Araque y Godoy, 2012). Las variables psicosociales relacionadas con el bienestar subjetivo que más impacto han tenido en la literatura han sido el optimismo (Ferreira y Sherman, 2007), las estrategias de afrontamiento (Caprara y Steca, 2005), la autoestima (García, Marín y Bohórquez, 2012), la autoeficacia (Caprara y Steca, 2005; Piquart y Sorensen, 2000) y el control percibido (Diener et al., 1999).

El bienestar subjetivo parece ser bastante estable a lo largo de la vida (Diener et al., 1999; Suh, Diener y Fujita, 1996; Suh, Diener, Oishi y Triandis, 1998), aunque se ha señalado la existencia de un patrón evolutivo en forma de U invertida (GodoyIzquierdo et al., 2012; Godoy-Izquierdo, Martínez y Godoy, 2009), de modo que a partir de los 40 años aproximadamente el bienestar subjetivo parece ser menor para mejorar alrededor de los 50 años y alcanzar posteriormente niveles semejantes a los de la mitad de la adultez (Blanchflower y Oswald, 2008; Godoy-Izquierdo et al., 2012).

En lo relativo a la vejez propiamente dicha, la visión tradicional ha asociado esta etapa de la vida con infelicidad y ausencia de bienestar subjetivo (Lang y Heckhausen, 2001; Lelkes, 2008; Mrockzek y Spiro, 2005), aunque en los últimos años diversos autores han considerado estos resultados inconclusos y no determinantes (Cartesen, Pasupathi, Mayr y Nesselroade,
2000; Ehrlich e Isaacowtiz, 2002; J.A. García (2011). Variables Psicosociales que inciden en la calidad de vida del alumnado participante en programas universitarios de mayores. Tesis Doctoral. Sevilla: Universidad de Sevilla).

Godoy-Izquierdo et al. (2012) indican que los principales predictores de bienestar subjetivo en la adultez tardía son la satisfacción con el estado de salud, el estatus marital, las relaciones sociales, la satisfacción de las necesidades básicas, las habilidades personales, la autoeficacia y el ocio activo. Partiendo de la importancia éstas variables -especialmente estado de salud y ocio activo-, la práctica de actividad física se postula como un elemento a tener en cuenta en la evaluación e intervención en bienestar en los mayores (Guillén, 1996; Menec, 2003; Pawlowsky, Downward y Rasciute, 2011).

La práctica de actividad física por parte de los mayores de 65 años ha sido relacionada una mayor calidad de vida (Rejeski, Brawley y Shumaker, 1996; Sánchez, Ureña y Garcés de los Fayos, 2002) llegando incluso a postularse que esta mejoría seguiría un patrón lineal, de modo que la mayor calidad de vida correspondería a sujetos con elevados niveles de práctica de actividad física (Jürgens, 2006). Para Elavsky et al. (2005), esta relación tendría no sólo efectos a corto plazo sino también a largo plazo extendiéndose durante un período temporal de un año. Por el contrario, el sedentarismo en personas mayores se ha relacionado con una disminución de sus capacidades físicas, cognitivas y psicológicas (Guillén, 1996; Guillén y Sánchez, 2010).

La relación entre actividad física y calidad de vida está mediada, según diferentes autores (Márquez, Hu, Jerome y Diener, 2005; McAuley et al., 2006), por los beneficios consecuentes de la implicación activa en el ejercicio; del mismo modo es posible entender que la relación entre sedentarismo y disminución de la calidad de vida se encontraría mediada por los mismos mecanismos. Beneficios de la actividad física como la autoeficacia (Dionigi, 2007; McAuley, Blissmer, Katula, Duncan y Mihalko, 2000), la autoestima (García et al., 2012), el afecto positivo (Salmon, Owen, Crawford, Bauman y Sallis, 2005) o la satisfacción con la vida (Meléndez, Tomás, Oliver y Navarro, 2008) promocionarían el bienestar subjetivo o lo disminuirían ante su inexistencia/desaparición.

Mientras que la relación entre calidad de vida y/o bienestar subjetivo y la práctica de actividad física ha sido previamente abordada en la literatura, no ha sido posible encontrar estudios que relacionen felicidad y ejercicio. Así, el objetivo de este estudio fue explorar la felicidad - presente y pasada- de las personas mayores de 65 años así como la relación que la práctica de actividad física pudiera tener con ella. De este objetivo general se desprendieron las siguientes hipótesis:

a) La felicidad presente será estimada menor que la felicidad pasada.

b) Los mayores que realizan actividad física estimarán mayor su felicidad que aquellos sedentarios.

c) La pérdida de actividad física implicará menores niveles de felicidad presente. 


\section{Método}

\section{Participantes}

La muestra -36 hombres y 68 mujeres- se formó por accesibilidad, contactando con personas mayores en diferentes espacios públicos. La edad media de los participantes fue 76.34 años $($ Mín. $=64$, Máx. $=91, D T=8.10)$.

En cuanto al historial de actividad física de los participantes 26 de ellos $(25 \%)$ siempre han estado involucrados en algún tipo de actividad física o deportiva, 8 de ellos (7.7\%) únicamente lo hicieron en el pasado, 44 realizan en esta etapa de su vida actividad física o deportiva por primera vez $(42.3 \%)$ mientras que 26 participantes (25\%) jamás se han involucrado en actividad física ya sea de manera formal o informal.

\section{Instrumentos}

Se desarrolló ex profeso una ficha de datos sociodemográficos que incluyó edad, género, estado civil, número de hijos, tipo de residencia, personas con las que se convive en el lugar de residencia, situación laboral, nivel académico e historial de actividad física y deporte.

Para la evaluación de los hábitos de actividad física/sedentarismo se empleó el Global Physical Activity Questionnaire -GPAQ- 2.0 en versión española (OMS, 2012). El GPAQ se compone de 16 ítems de los cuales 6 son de obligado cumplimiento mientras que los 10 restantes se cumplimentan en caso de respuestas afirmativas a preguntas llave. Este instrumento tiene en cuenta la actividad física realizada, su intensidad y el contexto en el que ésta se realiza (trabajo, desplazamiento $\mathrm{u}$ ocio); además, incluye una estimación del tiempo empleado en comportamientos sedentarios.

La evaluación de la felicidad y las fuentes de ésta se realizó mediante la Escala de Felicidad (Godoy-Izquierdo, Lara, Vázquez, Araque y Godoy, 2006). Esta escala se compone de 22 ítems que el sujeto ha de valorar en una rango 0-10. De estos ítems, 2 evalúan la felicidad actual y pasada y los 20 restantes la influencia de diferentes variables (como familia y relaciones familiares, hábitos y estilos de vida, herencia genética, etc.) en la felicidad actual de la persona.

\section{Procedimiento}

Se contactó con los mayores entrevistados en residencias de mayores, instalaciones deportivas municipales y diferentes zonas públicas como mercados, parques, calles céntricas transitadas, etc. Una vez realizada la presentación del entrevistador, se procedía a informar a los participantes de los objetivos del estudio, el formato de participación en el que se les deseaba involucrar y el tratamiento confidencial de sus datos. Tan sólo se entrevistó a aquellas personas que dieron su consentimiento y mostraron signos de adecuados estados de alerta y comprensión. Los cuestionarios se cumplimentaron en todas las ocasiones por un entrevistador entrenado que leía cada ítem al participante y procedía, cuando era necesario, a explicar las dudas que cada uno de ellos pudiera suscitar.
Análisis de datos. Los datos arrojados por las entrevistas fueron codificados y volcados en una matriz de SPSS 20.0 (IBM Corporation, 2012). De forma previa al análisis de datos se realizaron pruebas de normalidad y homocedasticidad de la muestra con el fin de tomar decisiones acerca de las pruebas estadísticas a emplear. Se determinó una distribución no normal de la muestra, así como la no homogeneidad de varianzas para las variables principales del estudio (pruebas de Kolmogorov-Smirnov y Levene, $p<.05$ ). Por ello se decidió utilizar pruebas no paramétricas en los análisis estadísticos realizados para este estudio. La primera hipótesis fue analizada mediante una prueba Wilcoxon de comparación de grupos dependientes, mientras que las hipótesis segunda y tercera se examinaron con pruebas Kruskall-Wallis para comparación de grupos independientes; para completar los análisis de la tercera hipótesis se efectuaron contrastes a posteriori Mann-Whitney.

\section{Resultados}

La Tabla 1 muestra los principales descriptivos de las variables objeto de estudio:

Tabla 1.

Descriptivos de las principales variables objeto de estudio.

\begin{tabular}{lccccc}
\hline \multicolumn{1}{c}{ Variables } & $N$ & Min. & Máx. & $M$ & $D T$ \\
\hline Feliz actualidad & 104 & .00 & 10.00 & 6.23 & 2.86 \\
Feliz pasado & 104 & 1.00 & 10.00 & 8.35 & 2.04 \\
Actividad física* & 104 & 1.00 & 4.00 & 2.67 & 1.26 \\
Historial de actividad física** & 104 & 1.00 & 4.00 & 2.50 & 1.03 \\
\hline
\end{tabular}

Nota: $* 1=$ sedentarismo, $2=$ en el pasado, $3=$ en el presente, $4=$ siempre. ** $1=$ nunca, $2=$ siempre, $3=$ pasado, $4=$ presente.

Con el objetivo de explorar la felicidad actual estimada por los participantes así como la felicidad pasada y la relación entre ambas, se realizaron pruebas no paramétricas de comparación de grupos dependientes. Los resultados mostraron diferencias significativas basadas en los rangos negativos de Wilcoxon $(Z=-6.02, p=.00)$ entre la felicidad actual y la pasada, siendo superior esta última.

Para indagar en la influencia de la actividad física realizada por los mayores de 65 años en el estado actual de felicidad se ejecutaron pruebas no paramétricas de comparación de grupos independientes. Los resultados, Tabla 2, indicaron diferencias significativas en la felicidad actual de los participantes en función de su implicación en tareas de ejercicio físico, estimando mayor su felicidad aquéllos que realizan actualmente algún tipo de actividad física.

Tabla 2.

Medias, desviaciones típicas y prueba $\chi^{2}$ para la "Felicidad Actual" en función de la actividad física realizada.

\begin{tabular}{lcccl}
\hline Actividad física realizada & $M$ & $D T$ & $X^{2}$ & $p$ \\
\hline Sedentarismo & 4.65 & 2.53 & 22.63 & .00 \\
Actividad física & 7 & 2.70 & & \\
\hline
\end{tabular}


Por otro lado, en lo relativo al historial de actividad física y su relación con la felicidad, se realizaron pruebas no paramétricas de comparación de grupos independientes. Los resultados mostraron diferencias significativas $(\chi 2=23.16, p=.00)$ en los niveles estimados de felicidad en función del historial de actividad física. Las Tablas 3 y 4 muestran respectivamente descriptivos e inferenciales para las comparaciones a posteriori entre las diferentes evoluciones en la implicación en actividades físicas o deportivas mostrando que: (I) los mayores que en la actualidad realizan actividad física son más felices que aquellos que la han realizado sólo en el pasado, nunca o siempre; (II) aquellas personas que siempre han realizado actividad física o aquellas que han comenzado a hacerla a partir de los 65 años son más felices que aquellos que la abandonaron en la vejez y (III) no haber realizado nunca actividad física está relacionado con menores niveles de felicidad actual que haberla realizado en algún momento de la vida o mantenerla.

Tabla 3.

Medias y desviaciones típicas para la "Felicidad Actual" en función del historial de actividad física.

\begin{tabular}{lccc}
\hline Historial de actividad física & $N$ & $M$ & $D T$ \\
\hline Pasado & 8 & 5.75 & .46 \\
Presente & 44 & 7.14 & 2.37 \\
Nunca & 26 & 4.31 & 2.81 \\
Siempre & 26 & 6.77 & 3.23 \\
\hline
\end{tabular}

Tabla 4.

Diferencias en "Felicidad Actual" entre grupos con distinto historial de actividad física. U de Mann-Whitney.

\begin{tabular}{|c|c|c|c|c|c|}
\hline Comparaciones & $N$ & $\begin{array}{c}\text { Suma de } \\
\text { rangos }\end{array}$ & $\begin{array}{l}\text { U Mann- } \\
\text { Whitney }\end{array}$ & $z$ & Sig \\
\hline $\begin{array}{l}\text { Nunca- } \\
\text { Siempre }\end{array}$ & $\begin{array}{l}26 \\
26\end{array}$ & $\begin{array}{l}501 \\
877\end{array}$ & 150 & -3.85 & $.00^{*}$ \\
\hline $\begin{array}{l}\text { Nunca- } \\
\text { Pasado }\end{array}$ & $\begin{array}{c}26 \\
8\end{array}$ & $\begin{array}{l}405 \\
190\end{array}$ & 54 & -2.12 & $.03 * *$ \\
\hline $\begin{array}{l}\text { Nunca- } \\
\text { Presente }\end{array}$ & $\begin{array}{l}26 \\
44\end{array}$ & $\begin{array}{c}587 \\
1898\end{array}$ & 236 & -4.12 & $.00^{*}$ \\
\hline $\begin{array}{l}\text { Siempre- } \\
\text { Pasado }\end{array}$ & $\begin{array}{c}26 \\
8\end{array}$ & $\begin{array}{c}515 \\
80\end{array}$ & 44 & -2.50 & $.01^{* *}$ \\
\hline $\begin{array}{l}\text { Siempre- } \\
\text { presente }\end{array}$ & $\begin{array}{l}26 \\
44\end{array}$ & $\begin{array}{c}925 \\
1560\end{array}$ & 570 & -.03 & .98 \\
\hline $\begin{array}{l}\text { Pasado- } \\
\text { Presente }\end{array}$ & $\begin{array}{c}8 \\
44\end{array}$ & $\begin{array}{c}116 \\
1262\end{array}$ & 80 & -2.47 & $.01 * *$ \\
\hline
\end{tabular}

\section{Discusión}

Las personas felices viven más y son más autónomas que las personas infelices (Danner, Snowdon y Friesen, 2001; Lucas, 2007), aunque esta relación está claramente mediada por la morbilidad prevalente y las conductas de salud como la práctica de actividad física (Castillo, Ortega y Ruiz, 2005; Godoy-Izquierdo et al., 2012). Así, el objetivo principal de este estudio fue explorar la felicidad -presente y pasada- de las personas mayores de 65 años así como la relación que la práctica de actividad física pudiera tener con ella. La primera hipótesis que se planteó en relación a este objetivo, predecía que la felicidad presente de los participantes sería menor que su felicidad pasada; los resultados permiten confirmar esa hipótesis al identificar una felicidad presente menor (en algo más de dos puntos en una escala Likert 0 a 10 respecto de las medias) que la pasada/evocada. Este decremento en la felicidad percibida es coherente con el mismo efecto encontrado por Godoy-Izquierdo et al. (2009, 2012) o Pinquart (2001), y que ha sido usualmente asociado con la pérdida de factores relacionados con la felicidad como la pareja (Haring-Hidore, 1985) o la salud (Danner et al. 2001; Lelkes, 2008). Aunque este hallazgo contradice a priori los postulados de la estabilidad de la felicidad a lo largo del ciclo vital (Diener, 1999; Diener y Suh, 1998; FernándezBallesteros, Zamarrón y Ruiz, 2001), las diferencias pueden relacionarse con las perspectivas de investigación adoptadas: mientras que las metodologías longitudinales y transversales no arrojan diferencias significativas en felicidad entre las diferentes edades e incluso identifican una mayor madurez emocional en la vejez que permite disfrutar más de la vida (FernándezBallesteros, 2004), la evaluación retrospectiva de la felicidad arroja mayores puntuaciones de felicidad pasada (Kennedy, Mather y Carstensen, 2004; Lacey, Smith y Ubel, 2006).

La segunda hipótesis postulaba que los mayores que realizan actividad física estimarían mayor su felicidad que los sedentarios, relación que se ve confirmada por los datos al estimar su felicidad en algo más de 2 puntos de media (en una escala 0 -10) para los mayores de 65 años que practican algún tipo de actividad física frente a aquellos que tienen un estilo de vida sedentario. Estos resultados confirman los encontrados por Rejeski et al. (1996), Sánchez et al. (2002), Guillén (1996) o Guillén y Sánchez (2010). Desde nuestro punto de vista, la relación felicidad-ejercicio es expresión de los beneficios de la actividad física sobre el bienestar físico, psicológico y social de la población general y de la población mayor en particular como ya indicaran, entre otros, Campos y Huertas (2003) o Capdevila (2005); en este sentido, la mejora de la condición física, las habilidades de autonomía, la auto-eficacia y las estrategias de afrontamiento podrían ser algunos de los productos de la actividad física más beneficiosos para un envejecimiento con bienestar subjetivo, aunque este aspecto debe ser comprobado en futuras investigaciones.

La última hipótesis proponía que la pérdida de actividad física implicaría menores niveles de felicidad presente de los mayores participantes. Los resultados muestran que:

a) Los mayores más felices son aquellos que practican actividad física en la actualidad, ya sea por una incorporación a la misma en esta etapa de su vida o por el mantenimiento de hábitos previos. Dos acercamientos explicativos, aunque no excluyentes, son posibles: por un lado la influencia positiva de la práctica de ejercicio físico (Huertas, 2003; Capdevila, 2005) y por otro lado el sedentarismo involuntario que obliga a algunos mayores, por su estado de salud física, a una inmovilidad forzosa.

b) Haber estado implicado en la práctica de actividad física en algún momento del ciclo vital (en el pasado, en el presente o de manera continua) está relacionado con un nivel mayor de 
felicidad que haber tenido un estilo de vida sedentario. Los mecanismos gracias a los cuales la práctica de actividad física tiene este papel protector de la felicidad están por determinar, aunque es posible plantear hipótesis como el aprendizaje y/o estimulación de habilidades de afrontamiento y manejo del estrés, la creación de redes extensas de apoyo social o la inclusión en estilos de vida saludables que permiten un envejecimiento saludable.

Entre las limitaciones de este estudio cabe destacar el reducido tamaño de la muestra, que no sólo resta representatividad a la misma sino que impide el uso de pruebas paramétricas de decisión estadística, en base a las cuales es posible explorar las relaciones planteadas con mayor precisión. Además, es necesario señalar la dificultad de la cumplimentación de las pruebas por parte de los participantes que, aunque asistidos por los investigadores, mostraron dificultades en la comprensión de algunos conceptos, en la limitación de su respuesta a las preguntas y en la utilización de escalas cuantitativas para la evaluación de conceptos cualitativos.

Futuras investigaciones deberían partir necesariamente de una adaptación de los instrumentos a la población mayor de 65 años y de una muestra de mayor tamaño. Además, replicar este estudio agrupando a los participantes en función de su voluntariedad/obligatoriedad en la práctica de actividad física permitiría clarificar el sentido de ocio u tratamiento que este ejercicio tuviera para los mayores y quizás correlacionarlo con la felicidad - presente y pasada- estimada. Por otro lado, una metodología longitudinal permitiría hacer un seguimiento estricto de las conductas de actividad física de los participantes y de su felicidad percibida, perspectiva que facilitaría confirmar el papel protector de la práctica de ejercicio en la felicidad de los mayores y, quizás, entender los mecanismos que median en dicha relación.

A pesar de las limitaciones señaladas y de las diferentes investigaciones que aún quedan por desarrollar, nuestros resultados permiten suponer un papel protector de la actividad física en el bienestar subjetivo de las personas mayores y por lo tanto de su felicidad. Este papel protector es fundamental en el diseño de los programas de promoción e intervención en el envejecimiento activo y positivo de la población.

\section{Referencias}

1. Blanchflower, D. G. y Oswald, A. J. (2008). Is wellbeing U-shaped over the life cycle? Social Science and Medicine, 66, 1733-1749. http://dx.doi.org/10.1016/j.socscimed.2008.01.030

2. Campos, J. y Huertas, F. (2003). Efectos de un programa de ejercicio físico sobre el bienestar psicológico de mujeres mayores de 55 años. Revista de Psicología del Deporte, 12, 7-26

3. Capdevila, L. (2005). Actividad Física y Estilo de Vida Saludable. Girona: Documenta Universitaria.

4. Caprara, G. V. y Steca, P. (2005). Affective and social self-regulatory efficacy beliefs as determinants of positive thinking and happiness. European Psychologist, 10, 275286. http://dx.doi.org/10.1027/1016-9040.10.4.275

5. Carstensen, L., Pasupathi, M., Mayr, U. y Nesselroade, J. R. (2000). Emotional experience in everyday life across the adult life span. Journal of Personality and Social Psychology, 79, 644-654. http://dx.doi.org/10.1037/00223514.79.4.644

6. Castillo, M. J.; Ortega, F. B. y Ruiz, J. (2005). Mejora de la forma física como terapia antienvejecimiento. Medicina Clínica (Barc),124, 146-155. http://dx.doi. org/10.1157/13071011

7. Danner, D. D., Snowdon, D. A. y Friesen, W. V. (2001). Positive emotions in early life and longevity: Findings from the nun study. Journal of Personality and Social Psychology, 80, 804-813. http://dx.doi.org/10.1037/00223514.80.5.804

8. Diener, E. (1984). Subjective well-being. Psychological Bulletin, 95, 542-575. http://dx.doi.org/10.1037/00332909.95.3.542

9. Diener, E. y Suh, E. M. (1998). Subjective well-being and age: An international analysis. En K. Schaie, K. Warner \& L.M. Powell (Eds.), Annual review of gerontology and geriatrics: Focus on emotion and adult development, 17, 304-324.

10. Diener, E.; Suh, E. M.; Lucas, R. E.; Smith, H. L. (1999). Subjective well-being: three decades of progress. Psychological Bulletin, 125, 276-302. http://dx.doi. org/10.1037/0033-2909.125.2.276

11. Dionigi, R. (2007). Resistance training and older adults' beliefs about psychological benefits: the importance of self-efficacy and social interaction. Journal of Sport and Exercise Psychology, 29, 723-746.

12. Ehrlich, B. S. e Isaacowitz, D. M. (2002). Does subjective well-being increase with age? Perspectives in Psychology, $5,20-26$.

13. Elavsky, S.; McAuley, E.; Motl, R. W.; Konopack; J. F.; Márquez, D. X.; Hu, L.; Jerome, G. J.; Diener, E. (2005). Physical Activity Enhances Long-Term Quality of Life in Older Adults: Efficacy, Esteem, and Affective Influences. Annals of Behavioral Medicine, 30, 138-145. http://dx.doi. org/10.1207/s15324796abm3002 6

14. Fernández-Ballesteros, R. (2004). La psicología de la vejez. Encuentros multidisciplinares, 6, 11-22.

15. Fernández-Ballesteros, R., Zamarrón, M. D. y Ruiz, M. A. (2001). The contribution of socio-demographic and psychosocial factors to life satisfaction. Aging and Society, 21, 25-43. http://dx.doi.org/10.1017/S0144686X01008078

16. Ferreira, V. M. y Sherman, A. M. (2007). The relationship of optimism, pain and social support to wellbeing in older adults with osteoarthritis. Aging \& Mental Health, 11, 89-98. http://dx.doi.org/10.1080/13607860600736166

17. García, A. J.; Marín, M. y Bohórquez, M. R. (2012). 
Autoestima como variables psicosocial predictora de actividad física en personas mayores. Revista de Psicología del Deporte, 21, 195-200.

18. Godoy-Izquierdo, D.; Lara, R.; Vázquez, M. L.; Araque, F. y Godoy, J. F. (2012). Correlates of Happiness Among Older Spanish Institutionalised and Non-Institutionalised Adults. Journal of Happiness Studies, 13, 24-52.

19. Godoy-Izquierdo, D., Martínez, A. y Godoy, J. F. (2009). Balance afectivo en hombres y mujeres: Implicaciones de la edad y el sexo. Revista Internacional de Psicología Clínica y de la Salud, 2, 299-320.

20. Guillén, F. (1996). Calidad de vida y actividad física en la vejez: una perspectiva psicosocial. En M. Navarro, E. Brito, J. M. García y J. A. Ruiz (Eds.), Programas de Actividad Física y deportiva para las personas mayores, 179198. Las Palmas de Gran Canaria: Exmo. Cabildo Insular de Gran canaria.

21. Guillén, F. y Sánchez, M. C. (2010). La intervención del psicólogo/a del ejercicio y el deporte en la mejora de la salud y la calidad de vida en poblaciones especiales. Apuntes de psicología, 28, 329-340.

22. Haring-Hidore, M.; Stock, W. A.; Okun, M. A. y Witter, R. A. (1985). Marital status and subjective well-being: a research synthesis. Journal of marriage and the family, 47, 947-958. http://dx.doi.org/10.2307/352338

23. IBM Corp. Released 2012. IBM SPSS Statistics for Windows, Version 20.0. Armonk, NY: IBM Corp.

24. Jürgens, I. (2006). Práctica deportiva y percepción de calidad de vida. Revista Internacional de Medicina y Ciencias de la Actividad Física y el Deporte, 6, 62-74.

25. Kennedy, Q., Mather, M. y Carstensen, L. L. (2004). The role of motivation in the age-related positivity effect in autobiographical memory. Psychological Science, 15, 208-214. http://dx.doi.org/10.1111/j.0956-7976.2004.01503011.x

26. Lacey, H. P., Smith, D. M. y Ubel, P. A. (2006). Hope I die before I get old: Mispredicting happiness across the adult lifespan. Journal of Happiness Studies, 7, 167-182. http:// dx.doi.org/10.1007/s10902-005-2748-7

27. Lang, F. R. y Heckhausen, J. (2001). Perceived control over development and subjective well-being: Differential benefits across adulthood. Journal of Personality and Social Psychology, 81, 509-523. http://dx.doi.org/10.1037/00223514.81.3.509

28. Lelkes, O. (2008). Happiness across the life cycle: Exploring age-specific preferences. European Centre for Social Welfare Policy and Research, Policy Brief, March, 2, 1-16.

29. Liang, J.; Lawrence, R. H. y Bollen, K. A. (1987). Race Differences in Factorial Structures of Two Measures of Subjective Well-being. The Journal of Gerontology, 42, 426-428. http://dx.doi.org/10.1093/geronj/42.4.426

30. Lucas, R. E. (2007). Long-term disability is associated with lasting changes in subjective well-being: Evidence from two nationally representative longitudinal studies. Journal of Personality and Social Psychology, 92, 717-730. http:// dx.doi.org/10.1037/0022-3514.92.4.717

31. Márquez, D. X.; Hu, L.; Jerome, G. J. y Diener, E. (2005). Physical Activity Enhances Long-Term Quality of Life in Older Adults: efficacy, esteem and affective influences. Annals of Behavioral Medicine, 31, 99-103.

32. Mckenzie, B. y Campbell, J. (1987). Race, socioeconomic status and subjective wellbeing of older Americans. The International Journal of Aging and Human Development, 25, 43-61. http://dx.doi.org/10.2190/K35G-MAWEMNBR-AH48

33. McAuley, E.; Blissmer, B.; Katula, J.; Duncan, T. E. y Milhako, S. L. (2000). Physical activity, self-esteem and selfefficacy relationships in older adults: a randomized controlled trial. Annals of Behavioral Medicine, 22, 131-139. http://dx.doi.org/10.1007/BF02895777

34. McAuley, E.; Konopack, J. F.; Motl, R. W.; Morris, K. S.; Doerksen, S. E. y Rosengren, K. R. (2006). Physical Activity and Quality of Life in Older Adults: Influence of Health Status and Self-Efficacy. Annals of behavioral Medicine, 31, 99-103. http://dx.doi.org/10.1207/s15324796abm3101_14

35. McMahan, E. A.; Estes, D. (2012). Age-Related Differences in Lay Conceptions of Well-Being and Experienced Well-Being. Journal of Happiness Studies, 13, 79-101. http://dx.doi.org/10.1007/s10902-011-9251-0

36. Meléndez, J. C.; Tomás, J. M.; Oliver, A. y Navarro, E. (2008). Psychological and physical dimensions explaining life satisfaction among the elderly: A structural model examination. Archives of Gerontology and Geriatrics, 48, 291-295. http://dx.doi.org/10.1016/j.archger.2008.02.008

37. Menec, V. H. (2003). The relation between everyday activities and successful aging: A 6-year longitudinal study. Journal of Gerontology. Series B, Psychological Sciences and Social Sciences, 58, S74-S82. http://dx.doi.org/10.1093/ geronb/58.2.S74

38. Mroczek, D. K. y Spiro, A. (2005). Change in life satisfaction during adulthood: Findings from the Veterans affairs normative aging study. Journal of Personality and Social Psychology, 88, 189-202. http://dx.doi.org/10.1037/00223514.88.1.189

39. Okun, M. A.; Stock, W. A.; Haring, M. J. y Witter, R. A. (1984). Health and Subjective Well-Being: A Meta-Analysis. The International Journal of Aging and Human Development, 19, 111-132. http://dx.doi.org/10.2190/QGJN0N81-5957-HAQD

40. Organización Mundial de la Salud. GPAQ: Global Physical Activity Questionnaire (versión 2.0 en español). Accedido 20 de septiembre de 2012. http://www.who.int/chp/steps/ instrument/es/index.html

41. Pawlowski, T., Downward, P. y Rasciute, S. (2011). Subjective well-being in European countries - On the age-specific impact of physical activity. European Review of Aging and Physical Activity, 8, 93-102. http://dx.doi.org/10.1007/ 
$\underline{\text { s11556-011-0085-X }}$

42. Pinquart, M. (2001). Age differences in perceived positive affect, negative affect, and affect balance in middle and old age. Journal of Happiness Studies, 2, 375-405. http:// dx.doi.org/10.1023/A:1013938001116

43. Pinquart, M. y Sorensen, S. (2000). Influences of socioeconomic status, social network, and competence on subjective well-being in later life: A meta-analysis. Psychology and Aging, 15, 187-224. http://dx.doi.org/10.1037/08827974.15.2.187

44. Pinquart, M. y Sorensen, S. (2001). Gender Differences in Self-Concept and Psychological Well-Being in Old Age. A Meta-Analysis. The Journals of Gerontology, series B, 56, 195-213. http://dx.doi.org/10.1093/geronb/56.4.P195

45. Rejeski, W. J.; Brawley, L. R. y Shumaker, S. A. (1996). Physical activity and health-related quality of life. Exercise and Sports Sciences Reviews, 24, 71-108. http://dx.doi. org/10.1249/00003677-199600240-00005

46. Salmon, J.; Owen, N.; Crawford, D.; Bauman, A. y Sallis, J. F. (2003). Physical Activity and sedentary behavior: a population-based study of barriers, enjoyment and preference. Health Psychology, 22, 178-188. http://dx.doi. org/10.1037/0278-6133.22.2.178

47. Sánchez, P. A.; Ureña, F.; Garcés de los Fayos, E. J. (2002). Repercusiones de un programa de actividad física gerontológica sobre la aptitud física, autoestima, depresión y afectividad. Cuadernos de Psicología del Deporte, 2, 57-73.

48. Suh, E., Diener, E. y Fujita, F. (1996). Events and subjective well-being: Only recent events matter. Journal of Personality and Social Psychology, 70, 1091-1102. http:// dx.doi.org/10.1037/0022-3514.70.5.1091
49. Suh, E., Diener, E., Oishi, S. y Triandis, H. (1998). The shifting basis of life satisfaction judgments across cultures: Emotions versus norms. Journal of Personality and Social Psychology, 74, 482-493. http://dx.doi.org/10.1037/00223514.74.2.482

50. VanPraag, Frijters y Ferrer-i-Carbonell (2003). The anatomy of subjective well-being. Journal of Economic Behavior \& Organization, 51, 29-49. http://dx.doi.org/10.1016/ $\underline{\mathrm{S} 0167-2681(02) 00140-3}$

51. Veenhomen, E. (2000). The four qualities of life. Ordering Concepts and Measures of the Good Life. Journal of Happiness Studies, 1, 1-34. http://dx.doi. org/10.1023/A:1010072010360

52. Waldinger, R. J., \& Schulz, M. S. (2010). What's love got to do with it? Social functioning, perceived health, and daily happiness in married octogenarians. Psychology and Aging, 25, 422-431. http://dx.doi.org/10.1037/a0019087

Fecha de recepción: 3 de marzo de 2013 Fecha de recepción de la versión modificada: 13 de mayo de 2013 Fecha de aceptación: 18 de junio de 2013 